\title{
Circulating microRNAs potentially associated with progression of castration-resistant prostate cancer
}

\author{
Elena Anatolevna Pudova \\ Laboratory of Postgenomic Research \\ EIMB RAS \\ Moscow, Russia \\ pudova_elena@inbox.ru
}

Anastasiya Andreevna Kobelyatskaya Laboratory of Postgenomic Research EIMB RAS

Moscow, Russia

kaa.chel@mail.

\author{
Boris Yakovlevich Alekseev \\ Urological department \\ FSBI NMRRC \\ Moscow, Russia \\ mnioi@mail.ru
}

\author{
Georgy Sergeevich Krasnov \\ Laboratory of Postgenomic Research \\ EIMB RAS \\ Moscow, Russia \\ gskrasnov@mail.ru
}

\author{
Kirill Mikhailovich Nyushko \\ Urological department \\ FSBI NMRRC \\ Moscow, Russia \\ kirandja@yandex.ru
}

\author{
Anna Victorovna Kudryavtseva \\ Laboratory of Postgenomic Research \\ EIMB RAS \\ Moscow, Russia \\ rhizamoeba@mail.ru
}

\begin{abstract}
Metastatic castration-resistant prostate cancer (mCRPC) is characterized by a high mortality rate due to its aggressiveness. In the field of modern oncourology, monitoring the activity of a disease for patients of this category during therapy is an important clinical problem, for the solution of which informative markers are needed. Circulating microRNAs can serve as such markers, which demonstrate diagnostic and prognostic potential in various types of cancer, including prostate cancer. The aim of this study is to search for circulating miRNAs of mCRPC patients plasma during therapy that are potentially associated with the progression of the disease, as well as to study the contribution of these miRNAs to the general progression mechanism. Based on high-throughput sequencing, a bioinformatic analysis was carried out with identification of the microRNA profile potentially associated with the progression of the disease ( $p$-value $<0.05$ ): miR-221-3p, miR99b-3p, miR-145-5p, miR-375-3p, miR-20a-5p, miR-21-5p, miR451a, miR-199a-5p, miR-183-5p, miR-let-7i-5p and miR-let-7f5 .
\end{abstract}

Key words - castration-resistant prostate cancer, progression, microRNAs, exosomes, sequencing, markers

\section{Introduction}

Metastatic castration-resistant prostate cancer (mCRPC) is a phase of the disease with increased aggressiveness and overall survival of patients up to three years [4]. The most important clinical problem is the monitoring of disease activity during ongoing therapy, namely, determining the moment of progression (inefficiency of the current therapeutic concept). The classic diagnostic marker, prostate-specific antigen (PSA), at this disease stage is not a reliable biomarker, as patients had visceral metastases without increasing PSA levels [5]. To solve this problem, reliable and informative noninvasive markers are needed. Currently, the search for such markers is taking place in the direction of microRNA profiling. The aim of our study is the profiling of plasma samples circulating miRNAs, in which an increase in PSA levels during therapy was noted, which is associated with treatment failure in this period and, accordingly, with the progression of the disease. The results of this study may be useful for identifying predictive markers with subsequent validation by qPCR, and will also contribute to a fundamental understanding of the progression mechanisms of mCRPC.

\section{Methods}

The study included patients diagnosed with mCRPC during therapy. Blood was taken before treatment and every month until the disease progresses. Blood sampling was carried out in vacutainers with EDTA, followed by centrifugation. Isolation of total RNA from exosomes was carried out from $1 \mathrm{ml}$ of blood plasma using a exoRNeasy Serum-Plasma Midi Kit (Qiagen, Germany), according to the manufacturer's protocol. MicroRNA libraries for subsequent high throughput sequencing were prepared using a NEBNext ${ }^{\circledR}$ Small RNA Library Prep Set for Illumina (New England Biolabs) according to the manufacturer's protocol. High throughput sequencing was performed on a NextSeq500 instrument. The quality control of the reads was performed using the FastQC program. To perform the subsequent bioinformatics data analysis, the miRge 2.0 pipeline was used. The analysis of the number of reads of data (counts per million; CPM) was performed using the edgeR package in the statistical environment $\mathrm{R}$. To normalize the data, the TMM (trimmed mean of M-values) method was used. Further, as part of the analysis of differential expression, a quasilikelihood F-test (QLF) was used to calculate the reliability of the results. The results were considered statistically significant with a QLF p-value $<0.05$. Next, we tried to identify the pathways most affected by differentially expressed (DE) miRNAs. For this purpose, we used the mirPath 3.0 (DIANA tools) web service and TarBase 7.0 data.

\section{Results}

As a result of the study, the following microRNAs were identified that were potentially associated with mCRPC progression: miR-221-3p, miR-99b-3p, miR-145-5p, miR375-3p, miR-20a-5p, miR-21- 5p, miR-451a, miR-199a-5p, miR-183-5p, miR-let-7i-5p and miR-let-7f-5p. According to various studies, the identified microRNAs demonstrate oncogenic function in various types of cancer, including mCRPC $[1,2,3,6]$. For identified microRNAs, mirPath has found 49 pathways with $p<0.01$, Among these pathways, we noted the following most oncologically significant pathways: proteoglycans in cancer, Hippo signaling pathway, cell cycle, pathways in cancer, focal adhesion, transcriptional misregulation in cancer, prostate cancer and TGF-beta signaling pathway. 


\section{ACKNOWLEDGMENT}

This work was financially supported by the RFBR, grant no. 17-29-06085. This work was performed using the equipment of EIMB RAS "Genome" center (http://www.eimb.ru/ru1/ckp/ccu_genome_c.php).

\section{REFERENCES}

[1] Garofalo M, Quintavalle C, Romano G, Croce CM, Condorelli G. miR221/222 in cancer: their role in tumor progression and response to therapy. Curr Mol Med. 2012 Jan;12(1):27-33. doi: $10.2174 / 156652412798376170$.

[2] Huang X, Yuan T, Liang M, Du M, Xia S, Dittmar R, Wang D, See W, Costello BA, Quevedo F, Tan W, Nandy D, Bevan GH, Longenbach S, Sun Z, Lu Y, Wang T, Thibodeau SN, Boardman L, Kohli M, Wang L. Exosomal miR-1290 and miR-375 as prognostic markers in castrationresistant prostate cancer. Eur Urol. 2015 Jan;67(1):33-41. doi: 10.1016/j.eururo.2014.07.035. Epub 2014 Aug 14.
[3] Lin HM, Castillo L, Mahon KL, Chiam K, Lee BY, Nguyen Q, Boyer MJ, Stockler MR, Pavlakis N, Marx G, Mallesara G, Gurney H, Clark SJ, Swarbrick A, Daly RJ, Horvath LG. Circulating microRNAs are associated with docetaxel chemotherapy outcome in castrationresistant prostate cancer. Br J Cancer. 2014 May 13;110(10):2462-71. doi: $10.1038 /$ bjc.2014.181.

[4] Mostaghel EA, Montgomery B, Nelson PS. Castration-resistant prostate cancer: targeting androgen metabolic pathways in recurrent disease. Urol Oncol. 2009 May-Jun;27(3):251-7. doi: 10.1016/j.urolonc.2009.03.016.

[5] Pezaro C, Omlin A, Lorente D., Rodrigues DN, Ferraldeschi R, Bianchini D, Mukherji D, Riisnaes R, Altavilla A, Crespo M, Tunariu $\mathrm{N}$, de Bono J, Attard G. Visceral disease in castration-resistant prostate cancer. Eur Urol. 2014 Feb;65(2):270-273. doi: 10.1016/j.eururo.2013.10.055.

[6] Zhu J, Wang S, Zhang W, Qiu J, Shan Y, Yang D, Shen B. Screening key microRNAs for castration-resistant prostate cancer based on miRNA/mRNA functional synergistic network. Oncotarget. 2015 Dec 22;6(41):43819-30. doi: 10.18632/oncotarget.6102. 